\title{
Syntactic and Semantic Specialization and Integration in 5- to 6-Year-Old Children during Auditory Sentence Processing
}

\author{
Jin Wang ${ }^{1}$, Mabel L. Rice ${ }^{2}$, and James R. Booth ${ }^{1}$
}

\begin{abstract}
Previous studies have found specialized syntactic and semantic processes in the adult brain during language comprehension. Young children have sophisticated semantic and syntactic aspects of language, yet many previous fMRI studies failed to detect this specialization, possibly due to experimental design and analytical methods. In this current study, 5- to 6-year-old children completed a syntactic task and a semantic task to dissociate these two processes. Multivoxel pattern analysis was used to examine the correlation of patterns within a task (between runs) or across tasks. We found that the left middle temporal gyrus showed more similar patterns within the semantic task compared with across tasks, whereas there was no difference in the correlation within the
\end{abstract}

\section{INTRODUCTION}

One model of brain development posits that there are agerelated increases in the interactivity between specialized cognitive functions as children mature and become more skilled (Sirois et al., 2008; Johnson, 2001). It has also been shown that many developmental disorders have delayed or atypical processes of interactive specialization (Johnson, 2011), which suggests the importance of these processes in brain development. Karmiloff-Smith (1998) pointed out that accurately characterizing typical development is the key to understanding developmental disorders. Therefore, studying brain specialization and integration in developing children is of importance both in terms of promoting our understanding of typical development and in terms of providing a neural reference to determine what is different in children with developmental disorders.

Friederici (2012) has proposed a language comprehension model, which argues for specialized semantic and syntactic processing in the adult brain during language comprehension. The proposal is that the opercular (BA 44) part of the left inferior frontal gyrus (IFG) is specialized in syntactic processing (e.g., Friederici, 2018; Zaccarella, Schell, \& Friederici, 2017), whereas the triangular (BA 45, BA 47) part of the left IFG and the left

\footnotetext{
${ }^{1}$ Vanderbilt University, ${ }^{2}$ University of Kansas
}

syntactic task compared with across tasks, suggesting its specialization in semantic processing. Moreover, the left superior temporal gyrus showed more similar patterns within both the semantic task and the syntactic task as compared with across tasks, suggesting its role in integration of semantic and syntactic information. In contrast to the temporal lobe, we did not find specialization or integration effects in either the opercular or triangular part of the inferior frontal gyrus. Overall, our study showed that 5- to 6-year-old children have already developed specialization and integration in the temporal lobe, but not in the frontal lobe, consistent with developmental neurocognitive models of language comprehension in typically developing young children.

middle temporal gyrus (MTG) are specialized in semantic processing (e.g., Goucha \& Friederici, 2015; Hagoort \& Indefrey, 2014; Binder, Desai, Graves, \& Conant, 2009). According to this model, the left posterior superior temporal gyrus (STG) integrates syntactic information from the opercular part of IFG with lexical-semantic information from the MTG (e.g., Zaccarella et al., 2017; Bornkessel, Zysset, Friederici, von Cramon, \& Schlesewsky, 2005). Although Friederici's (2012) language specialization model in adults is relatively well established, the evidence is lacking in terms of when the semantic and syntactic specialization for sentence-level processing emerges in the developing brain. Skeide and Friederici (2016) developed a model of language development, which suggests bottom-up processing for lexical-semantic and morphosyntactic categorization in the temporal lobe develops earlier whereas top-down processing for sentence-level semantics and syntax in the frontal lobe develops later and gradually from 3 years of age to young adulthood. They suggested that the functional selectivity for semantic information becomes neuroanatomically separable from functional selectivity for sentence-level syntactic information between the ages of 7 and 9 years. However, the relatively late emergence of functional selectivity for semantics and syntax in sentence processing might be due to the complexity of the sentences. Studying 5- to 6-year-old children processing simpler sentences may be a good age to determine when this language bttps://doi.org/10.1162/jocn_a_01477 
specialization emerges because these children are in the transition stage of mastering complex morphosyntactic principles as well as elaborating and refining their semantic representations (Conti-Ramsden \& Durkin, 2012; Kuhl, 2010).

A few studies have examined whether adult-like language specialization during sentence processing occurs in young children. Two studies used a single dissociation paradigm to explore the syntax-sensitive areas. In the Knoll, Obleser, Schipke, Friederici, and Brauer (2012) study, German children aged from 4;8 to 6;8 (years; months) were asked to listen to subject-initial sentences (e.g., [the dog $]_{\text {NOM }}$ kisses [the tiger $]_{\text {ACC }}$ NOM is the abbreviation for nominative, and ACC is the abbreviation for accusative) or object-initial sentences (e.g., [the tiger $]_{\text {ACC }}$ kisses [the dog $]_{\mathrm{NOM}}$ ) and perform a sentence-picture matching task (who does what to whom). They found that only children with higher language ability had greater activation in BA 45 for the object-initial compared with the subject-initial sentences. Because Friederici's (2002) model argues that BA 45 is associated with semantic processing whereas BA 44 is responsible for syntactic processing, this finding suggests that these children rely on semantic cues of the nominative (i.e., der) and accusative (i.e., den) markers to help complete tasks. Skeide, Brauer, and Friederici (2016) studied children aged 3-10 years as well as young adults. They designed sentences with a subject-relative clause or object-relative clause and also asked participants to do a sentence-picture matching task (who does what to whom). They found that adults, but not children, showed greater activation in the IFG opercular for sentences with object-relative versus subject-relative clause. In addition, both adults and 9- to 10-year-olds, but not 3- to 7-year-olds, exhibited greater activity in the posterior STG for sentences with object-relative versus subjectrelative clause. Together, these two studies suggest that young children aged 3-7 years have not yet developed adult-like brain activations in the left IFG opercular or the posterior STG during object-initial compared with subjectinitial sentences. Rather, these young children tend to rely on semantic regions to understand the more syntactically complicated sentences (e.g., the object-initial sentences). This tendency of young children to use semantic information is probably not only due to their limited syntactic ability but also to the experimental design. Children could take the semantic cues from the two morphological marker words (i.e., der, den) to judge the subject and the object of a sentence and complete the "who does what to whom" task. In other words, once the participants understand that the "der" means the subject whereas the "den" means the object, they could easily use the strategy of simply matching the noun word with a certain morphological marker ("der" or "den") to its role as either a "who" or "whom" to complete the task.

Because single dissociation studies cannot answer specialization questions due to the possible involvement of confounding factors, such as difficulty level, studies must use a double dissociation design to determine language specialization by manipulating both syntactic and semantic factors. These studies have also yielded inconsistent findings. Brauer and Friederici (2007) presented 5- to 6-year-old German children with sentences with a syntactic violation, in which the preposition was followed by a verb but not a correct noun phrase (e.g., The yogurt in-the tastes good); sentences with a semantic violation, in which a verb could not be integrated into context (e.g., The stone bleeds); as well as correct sentences (e.g., The iced-lolly melts). Children were asked to judge whether the sentence they heard was correct or not. They found that young children engaged areas in the left IFG (BA 44) for syntactically violated sentences compared with other types, which may reflect the early emergence of syntactic specialization in 5- to 6-year-old children. However, this effect could also be due to more effortful processing, as these sentences were more difficult than the others.

Skeide, Brauer, and Friederici (2014) used sentences with different levels of syntactic complexity (sentences with a subject-relative vs. object-relative clause) and different levels of semantic plausibility (plausible vs. nonplausible sentences by reversing the subject and the object in a sentence). They also asked participants to do a sentence-picture matching task (who does what to whom). They found that, in 3- to 4-year-old children, there was a significant interaction of syntactic and semantic processing in the left STG. In 6- to 7-year-olds, both the interaction and main effects of syntax and semantic processing were found in the left STG, but in different regions. In 9- to 10-year-old children, there was no interaction effect. There was a main effect of the syntactic factor in both the left IFG opercular and the IFG triangular, as well as a main effect of the semantic factor in the STG. These results generally support the idea that syntactic processing is gradually specialized from semantic processing. However, it does not fit well into Friederici's (2012) model that views STG as an integration area, IFG opercular as a syntactic area, and IFG triangular and MTG as semantic areas. This is probably because the experimental manipulations of both factors were confounded, making the main effects difficult to explain in terms of specialization. Children could judge the subject and the object of a sentence by using semantic cues from the two morphological marker words. Moreover, semantic plausibility heavily depended on the syntactical analysis of the subject and the object in a sentence.

Wu, Vissiennon, Friederici, and Brauer (2016) recruited 5- to 6-year-old children and also asked them to do a sentence-picture matching task. They designed a syntactic factor (subject-initial vs. object-initial sentences) as well as a semantic factor, animacy hierarchy, which included three levels of decreasing semantic plausibility: animate $_{\mathrm{NOM}}$-inanimate ${ }_{\mathrm{ACC}}$ (e.g., The donkey pushes the ball), animate ${ }_{\mathrm{NOM}}-$ animate $_{\mathrm{ACC}}$ (e.g., The tiger pulls the

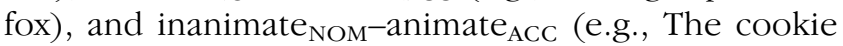


throws the fox). In this study, semantic plausibility relied little on syntactical analysis. They found an animacy hierarchy main effect in the IFG triangular, which suggests the emergence of semantic specialization in young children. They did not find regions specifically involved in syntactic processing, but this could be due to the confounding issues described above. Overall, the fMRI studies on young children aged approximately 5-6 years have demonstrated inconsistent results, with some studies finding syntactic sensitivity (e.g., Brauer \& Friederici, 2007) whereas others found semantic sensitivity (e.g., Wu et al., 2016) in the expected frontal areas, consistent with Friederici's (2012) language model. However, none of the studies have provided double dissociation results supporting semantic and syntactic specialization in young children.

Even though all previous fMRI studies failed to find a double dissociation of syntactic and semantic processing in 5- to 6-year-old children, ERP studies have reported an adult-like ERP pattern (N400) for semantic violations in 1- and 2-year-olds (Friedrich \& Friederici, 2005) and an adult-like ERP pattern (ELAN-P600) for phrase structure violations in 2.8-year-olds (Oberecker \& Friederici, 2006; Oberecker, Friedrich, \& Friederici, 2005). Therefore, the neural sensitivity to the semantics and syntax of language begins quite early in life. Skeide and Friederici's (2016) neurocognitive model of language development also suggested that, at the end of 3 years of life, children start to show sensitivity to single lexical items and phrase-level syntax, but they also argued the functional selectivity of semantic and syntactic processing develops gradually until young adulthood. Even though the brain specialization for semantically/syntactically complex sentences might occur as late as 7-9 years old as suggested by Skeide and Friederici's (2016) model, the specialization of semantics and syntax for simple sentence processing might emerge at the age of 5-6 years old as behavioral studies have consistently shown that the major semantic and syntactic aspects have been acquired by this age (Pinker, 1984; Gleitman \& Wanner, 1982).

The failure of finding a double dissociation in 5- to 6-year-olds in previous fMRI studies is likely due to two reasons. One reason is that experiment designs may have conflated semantic and syntactic processing. In the Knoll et al. (2012) study and others (Skeide et al., 2014, 2016; Wu et al., 2016), the manipulation of the syntactic or semantic factors may have been confounded when children tried to complete the sentence-picture matching task. In addition, Brauer and Friederici (2007) used anomalous sentences. Unlike ERPs, which have a high temporal resolution, using anomalous sentences in slow hemodynamic fMRI studies may smear the participants' initial response to an anomaly with subsequent processes as participants try to make sense of the sentences (Davis \& Rodd, 2011). Another reason for failing to find a double dissociation in 5- to 6-year-old children is the analytical method employed. All the fMRI studies summarized above used the conventional univariate fMRI analysis. This conventional method calculates the averages of activation across voxels to increase the signal to a particular condition, so it may wash out the voxels with informative, but weaker responses.

Multivoxel pattern analysis (MVPA) is a good way to avoid the univariate analysis drawback by treating each voxel as an informative feature to detect the similarities and differences in patterns of features between categories (Lewis-Peacock \& Norman, 2013). Very few language studies have used the MVPA method to examine syntactic and semantic specialization. Fedorenko, Nieto-Castañon, and Kanwisher (2012) designed four conditions of stimuli: sentences, word lists (e.g., "In because new robbery soon every angry directions tracy morning and battle"), jabberwocky (e.g., "After the bonter mellvered the perlen he mested to weer on colmition"), and nonword lists (e.g., "Was during cusariests fick prell pront the pome villpa and wronetist she"). The contrasts of sentences versus word lists and jabberwocky versus nonword lists were used to find the syntactic areas, whereas the contrasts of sentences versus jabberwocky and word lists versus nonword lists were used to find the semantic regions. Fedorenko et al. (2012) used the Haxby et al. (2001) approach, which correlates the patterns of activation within versus across categories. If the within-category correlations are higher than across-category correlations, the reasoning is that this area can distinguish the two categories. They found that many brain regions could discriminate between the conditions. All brain regions showed better discrimination in the semantic dimension than the syntactic dimension. No regions were found in which better discrimination occurred in the syntactic dimension. Based on these results, the authors then concluded that there are no specialized lexical and syntactic areas. However, the Fedorenko et al. (2012) results could mainly reflect differences in the degree to which the sentence-like stimuli are unfamiliar to the participants. Thus, studies with a more stringent design are needed to explore specialization of semantic and syntactic representations.

Yang, Marslen-Wilson, and Bozic (2017) used a syntactic complexity (word, short phrase, and long phrase) by frequency (low, mid, and high) design. They used representational similarity analysis (Kriegeskorte, Goebel, \& Bandettini, 2006), an MVPA method involving correlational matrices. They found that the left dorsal IFG (BA 44/BA 45) and posterior STG/MTG could differentiate words versus phrases along the syntactic dimension. The specialization of BA 44 for syntactic processing is consistent with Friederici's (2012) language model. However, BA 45 and MTG may have been sensitive to the manipulation of syntactic complexity because the comparison of words to long phrases also introduced semantic complexity. The MVPA analysis also showed a frequency effect of simple words in the left anterior ventral IFG (BA 47), possibly reflecting semantic accessibility, 
consistent with Friederici's (2012) model. Overall, the MVPA studies suggest that this method is sensitive in finding subtle representational differences between experimental conditions. However, there is no clear MVPA evidence to indicate whether the representational patterns of syntactic and semantic information are specialized in the brain.

In the current study, we used a semantic task involving a plausibility judgment and a syntactic task involving a grammaticality judgment to discern if 5- to 6-year-old children have syntactic and semantic specialization. By using separate tasks, we increased our chances of detecting the specialization for syntactic and semantic processes. Moreover, we only compared the grammatically correct sentences in the syntactic task with semantically plausible sentences in the semantic task, which allowed us to examine the comprehension processes of normal sentences rather than anomalous sentences. We used both univariate analysis and MVPA. We expected that the MVPA method would be more sensitive than the univariate analysis in detecting specialization in 5- to 6-yearold children. According to Friederici's (2012) language model, we hypothesized that if 5- to 6-year-old children have syntactic and semantic specialization, the left IFG opercular would show more similar patterns within the syntactic task than across tasks, whereas the left IFG triangular and MTG areas would show more similar activation patterns within the semantic task than across tasks. As for the left STG area, we hypothesized that it would be a semantic and syntactic integration area where the patterns of both within-semantic and within-syntactic tasks would be more similar than across tasks.

\section{METHODS}

\section{Participants}

Thirty-four children (mean age $=5.9$ years, range $5.5-$ 6.6 years, 19 girls) participated in the fMRI study. Children were recruited from the Austin, Texas, metropolitan area. Informed consent was obtained from the parents. The institutional review board approved all of the following procedures.

Participants were given developmental history questionnaires completed by their parents and a series of screening tests. The screening tests included a handedness interview in which the children were asked to pretend to write, draw, pick up, open, and throw something, as well as the Diagnostic Evaluation of Language Variation, Part 1 Language Variation Status (Seymour, Roeper, \& De Villiers, 2003). All the children met the following inclusionary criteria: (1) primarily right-handed, defined as performing at least three out of five items using their right hand; (2) mainstream English speakers, defined as scoring 7 or more (out of 15) for 5-year-olds and 8 or more (out of 15) for 6-year-olds on the main stream English items of the Diagnostic Evaluation of Language Variation; (3) no diagnosis of attention-deficit/hyperactivity disorder, neurological disease, psychiatric disorder, learning disability, or language disorder; and (4) normal hearing and normal or corrected-to-normal vision.

Standardized testing was then administered to assess their IQ and language abilities. This included the nonverbal subtest in the KBIT-2 (Kaufman \& Kaufman, 2004) and the core language measure in the Clinical Evaluation of Language Fundamentals (Wiig, Semel, \& Secord, 2013). All children had a standard score above 80 on both IQ and language ability.

\section{Experimental Procedure}

\section{Stimuli}

All sentence stimuli in the syntactic task and the semantic task had the following structure: An optional carrier phrase ("Last week"/"Every day") + subject and verb phrase (e.g., "She baked") + number and object (e.g., "two cakes"). The sentences included one of the following four verb forms: (1) third-person present tense (-s); (2) present progressive copula (be); (3) auxiliary verb (do); and (4) simple past tense (-ed). Each condition had five sentence stimuli for each verb form (see below for a description of conditions). Stimuli were matched across all conditions in each task in terms of the written word frequency (Masterson, Stuart, Dixon, \& Lovejoy, 2010; Balota et al., 2007), the number used (one/two/ three/four/five/six), the subject used (he/she/they), the number of syllables (6-8), and the frequency of "not" usage in the sentences.

\section{Syntactic Task}

In each trial, children heard one auditory sentence, presented binaurally through earphones. There were three conditions of sentence stimuli: grammatically correct (Gram), plurality violation (PVio), and finiteness violation (FVio; for examples, see Table 1). A carefully matched frequency-modulated white noise burst served as the auditory perceptual control (PC) condition. The children were asked, "does the way she speak sound right?" They were instructed to respond to all trials as quickly and accurately as possible, using their right index finger for a yes response in Gram and PC conditions and using their right middle finger for a no response in PVio and FVio conditions. Throughout the trial, a blue circle remained on the screen during the auditory stimuli presentation and turned yellow $1000 \mathrm{msec}$ before the trial ended to remind the participants to respond. The duration of each sentence was 2700-4500 msec. The duration of the response interval was $2300 \mathrm{msec}$. To optimize the extraction of the hemodynamic response, intertrial intervals of 0,575 , or $1150 \mathrm{msec}$ were added randomly in equal proportions, resulting in a duration of 5000-7950 msec for each trial. The length of trials was equated across conditions. The four conditions were pseudorandomized so 
Table 1. Auditory Stimuli Conditions and Examples

\begin{tabular}{lllll}
\hline Task & Condition & Response & Brief Explanation & Example \\
\hline Semantic task & SCon & Yes & Strongly congruent & Last week, she baked two cakes \\
& WCon & Yes & Weakly congruent & He does not break two glasses \\
InCon & No & Incongruent & They are bouncing one paper \\
PC & Yes & Pes & Grammatical & "Sh-Sh" \\
Syntactic task & Gram & No & Finiteness violation & He dropping one book \\
& FVio & No & Plurality violation & She is fixing two clock \\
& PVio & Yes & Perceptual control & "Sh-Sh" they play one game
\end{tabular}

that there were no more than five same responses in a row. There were 20 trials for each condition, totaling 80 trials evenly divided into two runs. Each run lasted around $4.5 \mathrm{~min}$. The three sentence conditions in the syntactic task were designed according to the following standards. The plurality violation condition was defined as the mismatch between the number and object by either adding an "s" or omitting an " $\mathrm{s}$ " in the object noun word. The finiteness violation condition was defined as the inconsistency between the subject and verb phrase by either adding an inflection or omitting an inflection/auxiliary verb. The two conditions with grammatical violations were carefully matched in terms of the number of additions and omissions. The grammatically correct condition was defined as sentences without grammatical errors.

\section{Semantic Task}

Similar to the syntactic task, in each trial, children heard one auditory sentence, presented binaurally through earphones. There were three conditions of the sentence stimuli: strongly congruent (SCon), weakly congruent (WCon), and incongruent (InCon; for examples, see Table 1). A carefully matched frequency-modulated white noise burst served as the auditory PC condition. The children were asked, "Does the way she speaks make sense?" They were instructed to respond to all trials as quickly and accurately as possible by using the right index finger for a yes response in SCon, WCon, and PC conditions and using the right middle finger for a no response in the InCon condition. The presentation procedure was the same as the syntactic task. There were 20 trials for each condition, totaling 80 trials evenly distributed in two runs. Each run lasted approximately $4.5 \mathrm{~min}$.

The three sentence conditions in the semantic task were designed according to the following standards. The two congruent conditions were based on the association strength values between the verb and the object, as defined in the University of South Florida Free Association Norms (Nelson, McEvoy, \& Schreiber, 1998). The SCon condition had an association of $0.28-0.81(M=0.41$, $S D=0.12$ ) between the verb and the object in the sentence. The WCon condition had an association of $0.02-0.19(M=0.11, S D=0.05)$ between the verb and the object in the sentence. In the InCon condition, the verb and the object in the sentence had no semantic association.

Participants who scored within an acceptable accuracy range and had no response bias were included in our analysis. Acceptable accuracy was defined as the accuracy of the PC condition being greater than $60 \%$. We used this above chance accuracy criterion to be more confident that the children were engaged in the task. The lack of response bias was indicated by the accuracy difference between the InCon and SCon conditions or the difference between the PVio and Gram conditions being lower than $40 \%$. Five participants were excluded from the fMRI analyses because of these criteria.

Before taking part in the fMRI scanning session, participants were required to complete a mock scan session. They performed the same task in the mock scanner to ensure that they understood the task and were acclimated to the scanner environment. Different stimuli were used in the mock and real scanning session. The real scanning took place within a month of the practice session.

\section{Data Acquisition}

Participants lay in the scanner with a response button box placed in their right hand. The participants viewed a screen via a mirror attached to the inside of the head coil. The visual dot was projected onto a screen to keep participants focused on the task so that they would respond in time. Participants wore earphones to hear the auditory stimuli, and two ear pads were used to attenuate the scanner noise. The two runs of a task were usually acquired within one session. If participants failed to finish certain runs, we invited them back a second time soon after the first session. Overall time-in-scanner for one session was less than $1 \mathrm{hr}$. 
Images were acquired using 3-T Siemens Skyra MRI scanner with a 64-channel head coil. The BOLD signal was measured using a susceptibility weighted single-shot EPI method. Functional images were acquired with multiband. The following scan parameters were used: echo time $=30 \mathrm{msec}$, flip angle $=80^{\circ}$, matrix size $=128 \times$ 128 , field of view $=256 \mathrm{~mm}$, slice thickness $=2 \mathrm{~mm}$ without gap, number of slices $=56$, repetition time $=$ $1250 \mathrm{msec}$, multiband acceleration factor $=4$. A highresolution, T1-weighted 3-D image was acquired. The following scan parameters were used: repetition time $=$ $1900 \mathrm{msec}$, echo time $=2.34 \mathrm{msec}$, matrix size $=256 \times$ 256 , field of view $=256 \mathrm{~mm}$, slice thickness $=1 \mathrm{~mm}$, number of slices $=192$.

\section{Data Analysis}

\section{Preprocessing}

The SPM12 toolbox (Statistical Parametric Mapping; www.fil.ion.ucl.ac.uk/spm) was used to analyze the data. First, all functional images were realigned to their mean functional image across runs. Then the anatomical image was segmented and warped to the pediatric tissue probability map template (Wilke, Altaye, Holland, \& CMIND Authorship Consortium, 2017) to get the transformation field. An anatomical brain mask was created by combining the segmentation products (i.e., gray, white and cerebrospinal fluid) and then applied to its original anatomical image to produce a skull-stripped anatomical image. After that, we coregistered the mean functional image and all functional images to the skull-stripped anatomical image. Then, all the functional images were normalized to the pediatric template by applying the transformation field to them.

We created the pediatric tissue probability map template by using CerebroMatic (Wilke et al., 2017), a tool that makes SPM12-compatible pediatric templates with user-defined age, sex, and magnetic field. We chose the unified segmentation parameters estimated from 1919 participants (downloaded from https:/www.medizin.unituebingen.de/kinder/en/research/neuroimaging/software/) described in the Wilke et al. (2017) study and defined ages as 5.5-8 years old with 1-month interval, two girls and two boys at each age interval with 3T scans, resulting in a sample size of 124 for our pediatric template.

Art-Repair (cibsr.stanford.edu/tools/human-brain-project/ artrepair-software.html) was used to correct for motion effect on brain signal. We identified outlier volumes, defined as those with volume-to-volume head movement exceeding $1.5 \mathrm{~mm}$ in any direction, head movement greater than $5 \mathrm{~mm}$ in any directions from the mean functional image or deviations of more than $4 \%$ from the mean global signal. Subjects having more than $10 \%$ or more than six consecutive outlier volumes in each run were excluded from the current study.

The Gram and the SCon conditions were chosen as the best contrast to explore the semantic and syntactic spe- cialization, because on the one hand, both conditions required the same response (pressing the yes button), excluding the possible confounding factor that different responses might induce different brain activation patterns; on the other hand, both conditions were correct sentences, avoiding the potential confusion in processing anomalous sentences. In this study, we chose the SCon condition in the semantic task as a condition of interest rather than the WCon condition, because the SCon condition is more natural and semantically predictable.

\section{Masks of Interest}

Four language masks of interest (i.e., the opercular and the triangular part of the left IFG, the left STG, and the left MTG) were chosen based on Friederici's (2012) language comprehension model, which suggested that the opercular part of the left IFG is associated with syntactic processing, the triangular part of the left IFG and the left MTG are associated with semantic processing, whereas the left STG is associated with integration of both semantic and syntactic information during language processing. The four language masks of interest were defined as the overlap between activation at group level and the four anatomical masks of interest selected by using the anatomical automatic labeling (AAL) atlas in the WFU PickAtlas tool (www.nitrc.org/ projects/wfu_pickatlas). Because the AAL atlas was based on the adult brain, we warped the T1 structure of the AAL atlas to our pediatric T1 template using AFNI's $3 \mathrm{dNwarp}$ nonlinear coregistration and then applied this transformation to the AAL atlas. In this way, anatomical atlas masks were aligned with our pediatric T1 template.

\section{Univariate Analysis}

Before univariate analysis, the preprocessed data were smoothed using a 6-mm isotropic Gaussian kernel. The outlier volumes were repaired by interpolation between the nearest nonoutlier volumes. Six motion parameters estimated in the realignment step were entered in the firstlevel modeling as regressors, and the repaired volumes were deweighted (Mazaika, Hoeft, Glover, \& Reiss, 2009).

Then, statistical analyses at the first level were calculated using an event-related design with eight conditions from both semantic and syntactic tasks as conditions of interest. A high-pass filter with a cutoff of $128 \mathrm{sec}$ and an SPM default artificial mask threshold of 0.5 was applied. All trials were included in the analysis and modeled using a canonical hemodynamic response function. Contrast maps were first created for Gram minus PC, SCon minus PC, (Gram minus PC) $>$ (SCon minus PC), and (SCon minus PC) $>$ (Gram minus PC) for each individual. Then we used one sample $t$ tests at the group level for each contrast. A conjunction analysis of (Gram minus PC) $>$ (SCon minus PC) and Gram minus PC was conducted to find brain regions that showed specialization for syntactic processing. A conjunction analysis of (SCon minus PC) > (Gram minus PC) 


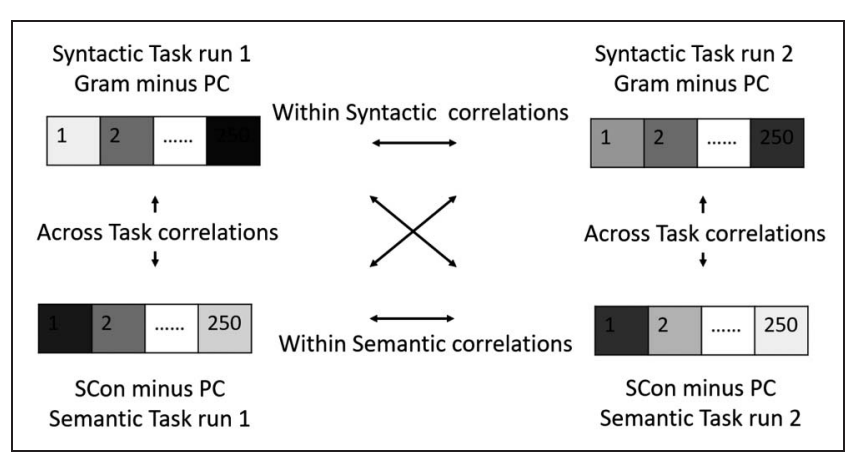

Figure 1. The MVPA in this study adopted from Haxby et al. (2001).

and SCon minus PC was also conducted to find brain regions that showed specialization for semantic processing. We did conjunction analyses rather than a simple contrast of (SCon minus PC) versus (Gram minus PC) to avoid finding effects that resulted from the deactivation from double subtraction, that is, greater activation for the PC. For all fMRI results reported in this article, a cluster was considered significant if it was greater than the cluster size threshold calculated with 3 dClustSim, at cluster-wise threshold $p<.05$ and voxel-wise threshold $p<.005$. A cluster size of 144 voxels was needed for significance for our four language masks of interest (i.e., the opercular and the triangular part of the left IFG, the left STG, and the left MTG, 12,190 voxels) for the analysis $(N=29$, autocorrelation function $[\mathrm{ACF}]=0.405,6.962,14.901)$. A cluster size of 648 voxels was needed for significance for the whole-brain level analysis.

Cluster significance was determined by using AFNI's 3dClustSim (February 2018) based on 10,000 iterations and the spatial ACF of mixed Gaussian and monoexponential form. We used the most recent version of 3dClustSim (February 2018), which no longer includes the bug that was considered to overestimate significance (fixed by AFNI in May 2015), following the suggestions made by Eklund, Nichols, and Knutsson's (2016) article regarding the inflated statistical significance achieved using some packages (i.e., SPM, FSL, and AFNI). Following these updates (Cox, Reynolds, \& Taylor, 2016), we used $3 d F W H M x$ to calculate the smoothness of the data for every single participant (as compared with previously used FWHMxyz values), using a spatial ACF, and then averaged those smoothness values across all participants. This averaged smoothness value was then entered into $3 \mathrm{dCl}$ CstSim to calculate the cluster size needed for significance for a given ROI. We included voxels significant at $p<.005$ uncorrected level and family-wise error corrected at $p<.05$ for significant clusters. The voxel size was $2 \times 2 \times 2 \mathrm{~mm}^{3}$.

\section{Multivoxel Pattern Analysis}

Unsmoothed data were used to do both feature selection and MVPA. Four language masks of interest (the opercular and the triangular part of the left IFG, the left MTG, as well as the left STG) were used. Within each mask, the top 250 most activated voxels in the contrast of all sentence conditions versus PC conditions across tasks were chosen as the features (voxels). Then, we extracted the beta value from each voxel for each trial using the least square single approach (Mumford, Turner, Ashby, \& Poldrack, 2012), a method for obtaining single trial betas in a fast event-related fMRI design (see script https://github.com/ritcheym/fmri misc/blob/master/generate_spm_singletrial.mc). To control for movement effect on brain signal, we also deweighted the outlier scans calculated by Art-Repair and included the six motion regressors in the model. After that, we calculated and compared the within-task and across-task correlations based on these top 250 voxels, similar analysis as described in the Haxby et al. (2001) study, to examine whether the brain could dissociate syntactic and semantic processes in our four masks of interest.

There were two runs (namely, run1 and run2) for each task in our study. Figure 1 shows the procedure of the MVPA conducted in the current study. Higher correlation means higher representational similarity in the brain. The within-semantic task correlation for each participant was calculated by correlating the averaged responses to SCon minus $\mathrm{PC}$ in the semantic task run1 to the averaged responses to SCon minus PC in the semantic task run2. In the same way, the within-syntactic task correlation for each participant was calculated by correlating the averaged responses to Gram minus PC in the syntactic task run1 to the averaged responses to Gram minus PC in the syntactic task run2. In addition, the averaged responses to Gram minus PC in either run1 or run2 of the syntactic task were correlated to the averaged responses to SCon minus PC in either run 1 or run 2 of the semantic task, resulting to four between-task correlations. The across-task correlation for each participant was calculated by averaging their four between-task correlations. We expected that the withinsyntactic correlations (but not within-semantic correlations) would be higher than across-task correlations in the hypothesized syntactic processing area, the opercular part of the left IFG; whereas the within-semantic correlations (but not within-syntactic correlations) would be higher than across-task correlations within the hypothesized semantic

Table 2. The Accuracies and RTs of Our Interested Conditions

\begin{tabular}{lcccc}
\hline Conditions & Accuracies (\%) & $T$ & $R T(\mathrm{msec})$ & $T$ \\
\hline Gram & $69.5(26.9)$ & $2.61^{*}$ & $3814(428)$ & $8.70 * *$ \\
SCon & $77.3(26.8)$ & & $3155(385)$ & \\
Syn_PC & $95.3(6.4)$ & -0.86 & $1281(843)$ & 0.87 \\
Sem_PC & $94.1(5.2)$ & & $1375(871)$ & \\
\hline
\end{tabular}

Syn_PC $=$ perceptual control for the syntactic task; Sem_PC $=$ perceptual control for the semantic task.

$* p<.05$
$* * p<.001$ 
Table 3. Brain Activations for the Syntax and Semantic Tasks

\begin{tabular}{|c|c|c|c|c|c|c|c|}
\hline \multirow[b]{2}{*}{ Region } & \multirow[b]{2}{*}{$L / R$} & \multirow[b]{2}{*}{ Brodmann's Area } & \multicolumn{3}{|c|}{ Coordinates } & \multirow[b]{2}{*}{ Voxels } & \multirow[b]{2}{*}{$Z$} \\
\hline & & & $x$ & $y$ & $z$ & & \\
\hline \multicolumn{8}{|c|}{ Within 4 Language Masks of Interest (Voxels > 144) } \\
\hline \multicolumn{8}{|l|}{ Gram_vs_PC } \\
\hline STG & $\mathrm{L}$ & 22 & -62 & -12 & 4 & 2548 & 6.82 \\
\hline \multicolumn{8}{|l|}{ SCon_vs_PC } \\
\hline STG & $\mathrm{L}$ & 22 & -62 & -12 & 2 & 1838 & 6.65 \\
\hline \multicolumn{8}{|c|}{ Whole Brain Analysis (Voxels > 648) } \\
\hline \multicolumn{8}{|l|}{ Gram_vs_PC } \\
\hline STG & $\mathrm{R}$ & 22 & 62 & -8 & 4 & 3196 & 7.08 \\
\hline STG & $\mathrm{L}$ & 22 & -62 & -12 & 4 & 3249 & 6.82 \\
\hline Fusiform area & $\mathrm{L}$ & 37 & -24 & -42 & 14 & 810 & 5.10 \\
\hline \multicolumn{8}{|l|}{ SCon_vs_PC } \\
\hline STG & $\mathrm{R}$ & 22 & 62 & -6 & 0 & 2256 & 6.96 \\
\hline STG & $\mathrm{L}$ & 22 & -62 & -12 & 2 & 2309 & 6.65 \\
\hline
\end{tabular}

related regions, namely, the triangular part of the left IFG and the left MTG.

For visualization of the top 250 most activated voxels across participants, BrainNet Viewer (Xia, Wang, \& He, 2013) was used to plot the voxels that were overlapped among at least five participants. The top 200 or top 300 most activated voxels during the sentence conditions compared with the PC conditions across tasks were also used as features to determine the reliability of the analyses. The results of significance tests were the same as when using the top 250 most activated voxels.

\section{RESULTS}

\section{Behavioral results}

Table 2 shows the means and standard deviations of the accuracies and RTs from our conditions of interest. For accuracies, the Gram condition in the syntactic task showed significantly lower accuracies than the SCon condition in the semantic task, $t(28)=2.61, p<.05$. No difference was found in the PC conditions for the syntactic task and the semantic task. For RTs, the Gram condition in syntactic task showed significantly longer RTs than the SCon condition in semantic task, $t(28)=8.70, p<.001$. The RTs of the PC conditions in the syntactic and semantic tasks showed no difference. These results show participants performed equally well in both tasks in PC conditions. However, grammatical judgment was significantly more difficult than semantic judgment for these 5- to 6-year-old children.

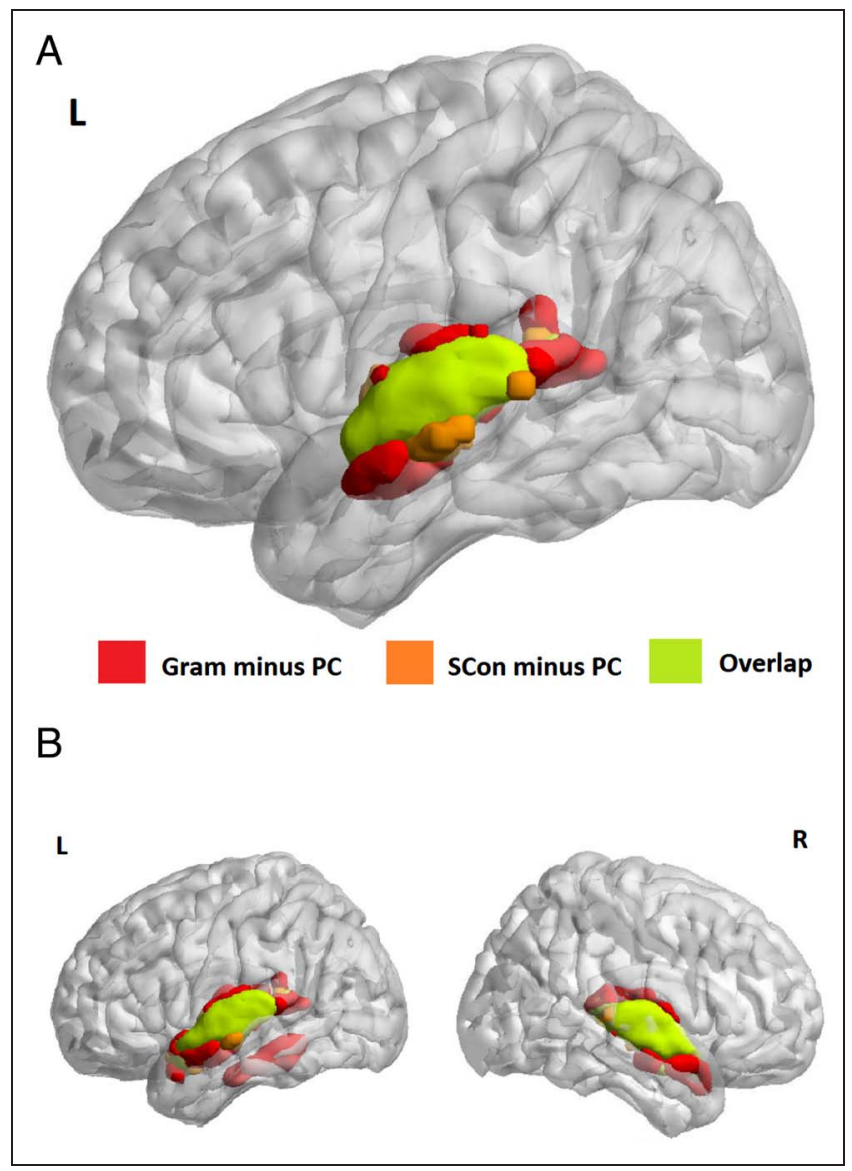

Figure 2. Brain activation maps for the syntactic and semantic tasks and their overlap within four language masks of interest (A) and at the whole-brain level analysis (B). 
Figure 3. MVPA results in the left frontal areas and temporal areas. (Left) The overlap voxels (features) among at least five participants within the four masks of interest: the opercular part of the left IFG (IFG opercular), the triangular part of the left IFG (IFG triangular), the left STG, and the left MTG (Right) The within-syntactic task correlations (Gram), within-semantic task correlations (SCon), and across-task correlations (Across). (A) Top: the results from IFG opercular and IFG triangular. (B) Bottom: The results from STG and MTG. Significance level: $* p<.05$, $* * p<.01, * * * p<.001$, Bonferroni corrected for multiple comparisons.
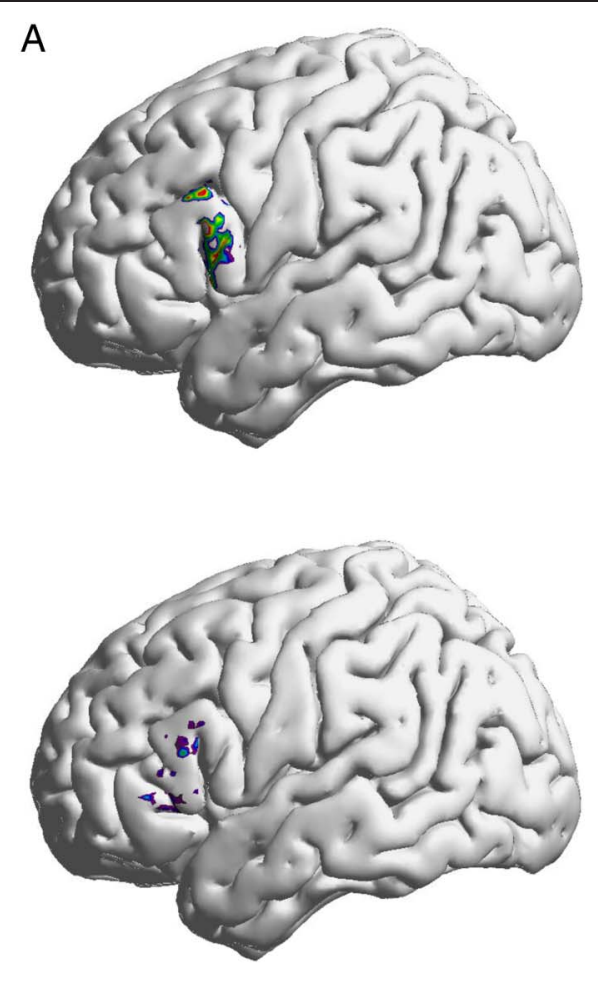

5
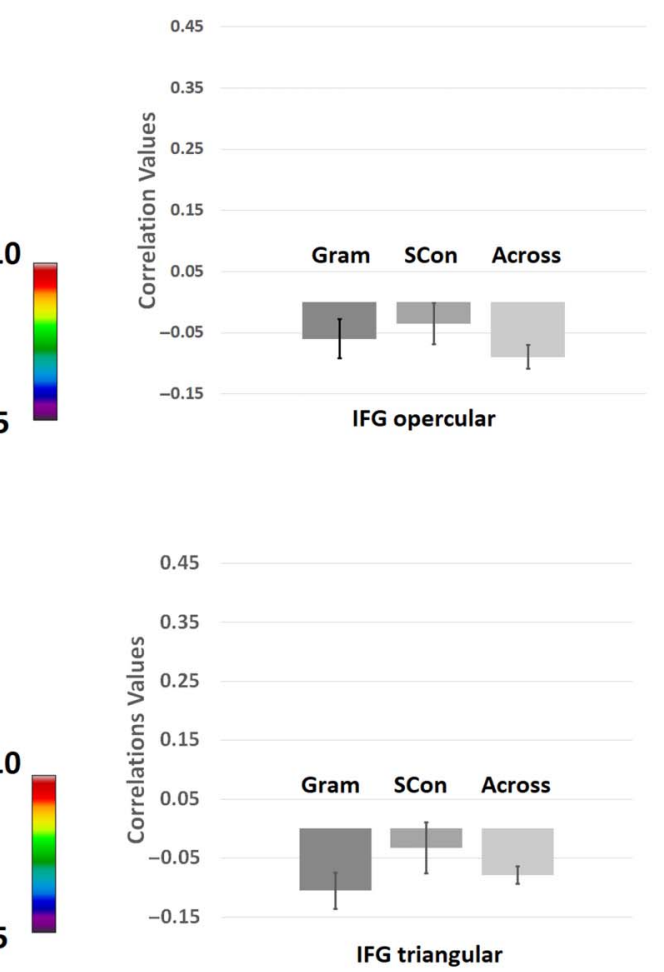

B

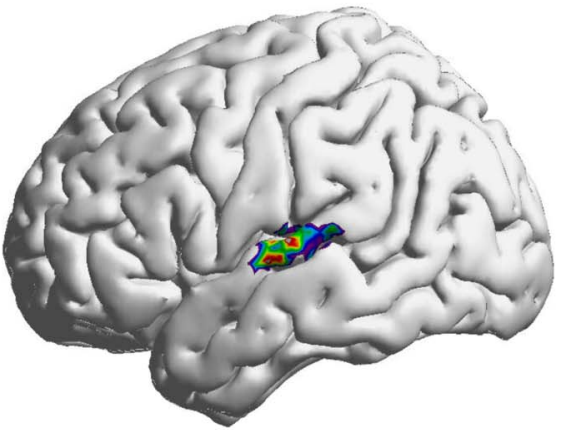

10
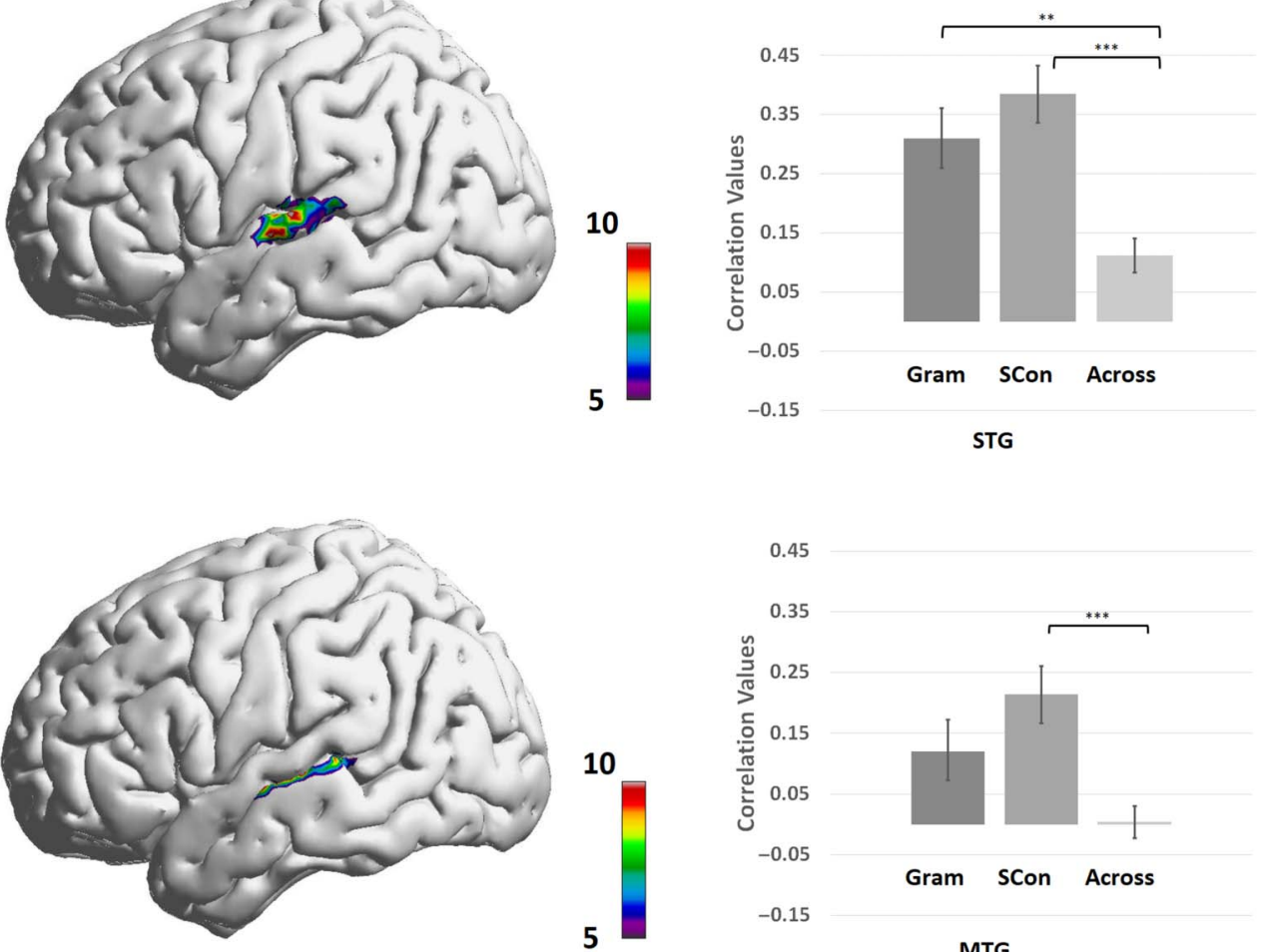

10

5
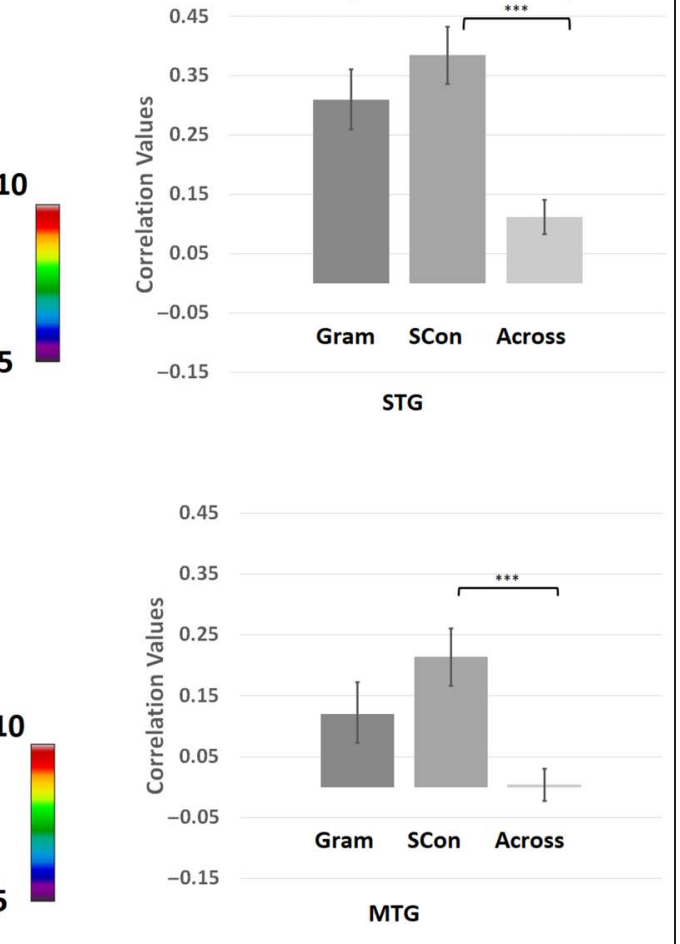


\section{Univariate Analysis Results}

Table 3 and Figure 2 shows the activation maps of Gram minus PC and SCon minus PC and their overlaps within our four masks of interest, that is, IFG opercular, triangular, MTG, and STG and at the whole-brain level. The left STG was significantly activated in both Gram minus PC and SCon minus PC within our four masks of interest, and they were largely overlapping. At the whole-brain level analysis, the left fusiform area was also significantly activated for Gram minus PC. However, the conjunction analysis of (Gram minus PC) > (SCon minus PC) with Gram minus PC showed no significant clusters both within our four masks of interest or at the whole-brain level. Similarly, the conjunction analysis of (SCon minus PC) $>$ (Gram minus PC) with SCon minus PC also showed no significant clusters. These results suggested that there was no semantic and syntactic specialization found in terms of overall brain activation.

\section{MVPA Results}

Figure 3A shows the results in frontal areas. On the left panel, it shows the overlapped voxels for at least five participants within the mask of IFG opercular and the IFG triangular areas. On the right panel, it shows within-syntactic (Gram) correlations, within-semantic (SCon) correlations, and across-task correlations. Two-way repeated-measures ANOVA for Level (within the syntactic task, within the semantic task, and across tasks) $\times$ Region (the opercular part of IFG vs. the triangular part of IFG) was conducted. There were no significant main effects or interactions, suggesting that no specialization occurred in the frontal region.

Figure 3B shows the representational similarity results in temporal areas. On the left panel, it shows the overlapped voxels for at least five participants within the mask of STG on the top and MTG on the bottom. On the right panel, it shows within-syntactic (Gram) correlations, within-semantic (SCon) correlations, and acrosstask correlations. Two-way repeated-measures ANOVA for Level (within the syntactic task, within the semantic task, and across tasks) $\times$ Region (MTG vs. STG) was also conducted. We found a significant main effect for Region, $F(1,28)=31.44, p<.001$, and a significant main effect for Level, $F(2,56)=17.00, p<.001$. We also found a significant interaction effect, $F(2,56)=3.43, p<.05$. Post hoc test showed that the STG showed overall more similar patterns than MTG $(p<.001)$ and that the withinsyntactic task and within-semantic task correlations were significantly higher than across tasks $(p<.05$ and $p<$ .001). Because of the significant interaction, one-way ANOVA was then conducted to determine the functional selectivity in each region. We found that, in STG, there were significant differences among the three levels, $F(2,56)=13.54, p<.001$. Post hoc tests showed that the within-syntactic correlations (Gram) were significantly higher than the across-task correlations in STG $(p<.001$, Bonferroni corrected) and the within-semantic correlations (SCon) were also significantly higher than the across-task correlations ( $p<.01$, Bonferroni corrected). No difference was found between within-syntactic correlations (Gram) and within-semantic correlations (SCon). These findings suggest that the left STG is an integration area that shows sensitivity to both semantic and syntactic information. In contrast, in the left MTG area, there were significant differences among the three levels, $F(2,56)=$ 8.63, $p<001$. Post hoc test showed that only the withinsemantic correlations (Scon) were significantly higher than the across-task correlations $(p<.001$, Bonferroni corrected), whereas there was no difference between withinsyntactic correlations (Gram) and across-task correlations, suggesting that the left MTG is specialized for semantic processing rather than syntactic processing.

\section{DISCUSSION}

This study explored whether the semantic and syntactic specialization during sentence-level processing emerges in young children by using simple sentences and a sensitive analytical method, the MVPA. We found both specialization and integration in the temporal lobe. The left MTG, as a hypothesized semantic region, showed more similar patterns within the semantic task than across tasks, whereas there was no difference between patterns within the syntactic task and across tasks, suggesting the MTG is specialized for semantic processing. The left STG, as a hypothesized integration region, showed more similar patterns within both the semantic task and the syntactic task as compared with across tasks. In addition, no difference was found between the pattern similarities within the semantic task and within the syntactic task, suggesting the STG is sensitive to both semantic and syntactic information. In contrast to the temporal lobe, we did not find any evidence for specialization or integration in the frontal lobe. Overall, our results suggest that children at 5-6 years old have only developed specialization and integration in the temporal lobe, but that the frontal lobe is slower to mature.

Our study found that the left MTG is specialized for semantic information in 5- to 6-year-old children. This specialization was indicated by more similar patterns within the semantic task as compared with across tasks and no difference between the patterns within the syntactic task versus across tasks. This finding is consistent with previous meta-analysis studies on adults (Hagoort \& Indefrey, 2014; Binder et al., 2009). Binder et al. (2009) found that the left MTG area is consistently involved in semantic processes, especially in language tasks. Hagoort and Indefrey (2014) found that the left middle and posterior MTG is consistently activated in highly demanding semantic compared with syntactic conditions.

Despite the fact that previous studies on young children aged 5-6 years old did not find such a semantic 
specialization effect in the left MTG (Wu et al., 2016; Brauer \& Friederici, 2007), we showed this semantic specialization in MTG in young kids for at least three possible reasons. First, we designed separate tasks so that children could focus their attention on either the semantic or the syntactic structure of the sentences, allowing us to unconfound these factors. Second, only correct sentences (i.e., Gram and SCon) were chosen as the conditions of interest, avoiding the use of anomalous sentences that employ other processes (Davis \& Rodd, 2011). Third, we used a MVPA, which may be a more sensitive way to detect the slight differences among experimental conditions (Lewis-Peacock \& Norman, 2013). By using a more stringent experimental design and a more sensitive analytical method, our research demonstrates semantic specialization in the brain activity of 5- to 6-year-old children in the left MTG.

In terms of integration, we found that within the left STG area, the language-sensitive voxels showed more similar patterns both within the semantic task and within the syntactic task compared with across tasks. Moreover, there was no difference in the similarity of the patterns within each of the tasks. This suggests a role of the left STG in the integration of both semantic and syntactic information during sentence comprehension, as suggested in Friederici's (2012) language model. The left STG has often been found to be activated together with the IFG opercular area in older children and adults when participants encounter syntactically more complex sentences compared with simple sentences (e.g., Skeide et al., 2016) or in the contrast of sentences versus word lists (e.g., Zaccarella et al., 2017). These findings appear to reflect the role of the left STG in merging syntactical hierarchies with lexical information. However, its functioning appears to go beyond syntactic processing. Many studies have found that STG is sensitive to the thematic role assignment (den Ouden et al., 2012) or semantic predictability (Dikker, Silbert, Hasson, \& Zevin, 2014; Obleser \& Kotz, 2010), suggesting its involvement in semantic processing. Zaccarella et al. (2017) also found that there was a white matter tract connecting the IFG opercular and the left STG, providing structural evidence supporting lexical syntactic integration in this area. Moreover, previous fMRI studies on young children directly comparing semantic and syntactic experimental conditions found no difference (Wu et al., 2016; Brauer \& Friederici, 2007) or interactive effects of semantic and syntactic processes in the STG (Skeide et al., 2014). Our finding that the STG is equally sensitive to both semantic and syntactic information is broadly consistent with these previous fMRI studies on young children.

Even though young children aged 5-6 years old have acquired major semantic and syntactic knowledge (Pinker, 1984; Gleitman \& Wanner, 1982), our results show that they do not show adult-like specialization in the opercular and triangular part of the IFG. This is in alignment with Skeide and Friederici's (2016) neurocognitive model of language development, which suggests bottom-up lexical and morphosyntactic processing in the temporal lobe develops earlier whereas top-down processing of semantics and syntax at sentence-level processing in the frontal lobe develops later. According to this model, the specialization of the frontal lobe in young adults during sentence-level processing plays a decisive role in reaching full efficiency of semantic and syntactic processing. This model predicts that the functional selectivity for sentence-level semantic information becomes neuroanatomically separable from syntactic information between the ages of 7 and 9 years, and only after the age of 10 years does BA 44 reaches its full specificity in processing complex syntax. Even though we used simpler sentences, we did not find evidence for sentence-level semantic and syntactic specialization in the frontal region, consistent with the prediction by Skeide and Friederici (2016).

As shown by the maps within our four masks of interest (see Figure 3, left), the top 250 voxels were distributed rather than clustered across participants, suggesting individual differences for language-sensitive voxels. These individual differences are likely the reason why it is difficult to find significant clusters showing specificity using univariate analysis at the group level. Young children are still developing so they might have greater individual differences of language-sensitive regions compared with older children and adults, and therefore, a more sensitive approach might be needed to detect functional specificity. Previous studies have shown that selecting languagesensitive regions by function within each individual participant improves the sensitivity of finding specificity effects (Fedorenko, Hsieh, Nieto-Castañón, WhitfieldGabrieli, \& Kanwisher, 2010). Therefore, before performing an MVPA, we chose language-sensitive features in four masks of interest within each individual participant.

The Rodd, Vitello, Woollams, and Adank (2015) metaanalysis focusing on the contrast between semantic and syntactic processing in adults found results that do not fit into Friederici's (2012) model. They found that the dorsal left IFG (BA 44, BA 45) was sensitive to both semantic and syntactic processing. They also found that the mid STG was only sensitive to semantic processing, whereas the posterior MTG was only sensitive to syntactic processing. Their discrepant findings may be due to their inclusion criteria. They included studies requiring either syntactic or semantic processing but not necessarily studies directly contrasting the two processes. McNorgan, Chabal, O'Young, Lukic, and Booth (2015) pointed out that only studies using direct contrasts should be included in meta-analysis because activation areas of spatial distributions associated with processing each type are more likely to be obscured by heterogeneous baseline. Another criterion of the Rodd et al. (2015) meta-analysis is that they chose studies regardless of the length of the language stimuli used, ranging from words to narratives. By using contrasts to dissociate semantic and syntactic processing at the sentence level, our study showed both specialization and integration in the temporal 
lobe, supporting the language comprehension model proposed by Friederici (2012) and the neurocognitive model of language development proposed by Skeide and Friederici (2016).

In terms of the results from the univariate analysis, our study did not find a semantic and syntactic specialization. This is broadly consistent with previous fMRI studies on young children, which did not show a double dissociation (Skeide et al., 2014, 2016; Wu et al., 2016; Knoll et al., 2012; Brauer \& Friederici, 2007). We found that the left STG was strongly activated during auditory sentence processing in both the semantic and the syntactic tasks, and they were largely overlapping. This is consistent with many previous studies, which showed activation in bilateral STG areas during auditory sentence comprehension (see review in Price, 2012). We also found that the left fusiform area was significantly activated for the syntactic task. This area is known as the basal temporal language region from many studies on aphasia patients (e.g., Sharp, Scott, \& Wise, 2004), which argues that it is associated with retrieval of meanings for speech comprehension. However, we found no significant difference between the semantic and syntactic tasks, suggesting that young kids may rely on semantics to help with processing in the more difficult syntactic task.

Our behavioral results showed that young children experienced more difficulty in grammatical judgment than semantic judgment. This is as expected from previous studies that documented that one particular property of English grammar, which is the requirement of finiteness on verbs, may not be fully adult-like in 5- to 6-year-old children (Rice \& Wexler, 2001; Tager-Flusberg \& Cooper, 1999). In our syntactic task, we included many sentences with finiteness violations. Children should be aware of the grammaticality in verb forms during the syntactic task, thus making it more difficult as this aspect of grammatical knowledge in 5- to 6-year-old children develops later in time. In our brain activation analysis, we incorporated behavioral performance in our neuroimaging analyses and found that this did not affect the results. We found that the general linear model results of brain activation for different contrasts were similar after controlling for accuracy. In addition, we found no significant correlations between the accuracy and the brain activation pattern similarities. These additional analyses suggest that our brain results were not merely reflecting the effect of task difficulty.

Our study not only contributes to a theoretical understanding of the language comprehension model proposed by Friederici (2012) and the neurocognitive model of language development as suggested by Skeide and Friederici (2016), it also has important implications for studies on developmental language disorders. Previous research found that children with specific language impairment are 1-2 years behind typical children in semantic and syntactic tasks (e.g., Rice, 2012). Mayes, Reilly, and Morgan (2015) reviewed previous neuroimaging studies on developmental language disorders, most of which were conducted on school-aged children. They found that children with language impairments generally showed atypical brain structure and function within core language regions such as the left IFG opercular and the left posterior STG, even though the difference (increase or decrease) was variable across studies. However, neuroimaging studies are lacking in preschool children with developmental language disorders under age 7 years (Morgan, Bonthrone, \& Liégeois, 2016). Only two studies focused on children younger than 7 years old. Dibbets, Bakker, and Jolles (2006) found that 6-year-old children with specific language impairment showed more activation in frontal and cingulate areas in an executive functioning task, suggesting a compensation mechanism. Kim et al. (2006) found that 3- to 6-year-old children with language impairment showed reduced white matter integrity in corpus callosum, suggesting a late neural myelination. By using language tasks, our study showed that both specialization and integration in the temporal lobe for sentence-level processing emerges in typical children as early as 5-6 years old. Future studies are needed in young children with language disorders to determine if there are associations between brain regions and markers of language impairment that persist longitudinally, suggesting a causal connection in brain function and language impairment. In addition, our results form a preliminary foundation for future longitudinal studies to test how this language specialization and integration in the temporal lobe during sentence processing predicts later semantic and syntactic specialization in the frontal lobe.

To sum up, by using simple sentences and MVPA, our study provided fMRI evidence showing that 5- to 6-yearold children have already developed both specialization and integration at the sentence level in the temporal lobe but have not yet developed adult-like processing in the frontal lobe. The results of this current study not only contribute theoretically to the neurocognitive model of language development proposed by Skeide and Friederici (2016) but also informs future studies in developmental language disorders, which might show lack of specialization and integration in the temporal lobe.

\section{Acknowledgments}

This work was supported by National Institutes of Health grant R01 DC013274 to James R. Booth.

Reprint requests should be sent to Jin Wang, Department of Psychology and Human Development, Vanderbilt University, Nashville, TN 37212, or via e-mail: jin.wang@vanderbilt.edu.

\section{REFERENCES}

Balota, D. A., Yap, M. J., Cortese, M. J., Cortese, M. J., Hutchison, K. A., Kessler, B., et al. (2007). The English lexicon project. Behavior Research Methods, 39, 445-459.

Binder, J. R., Desai, R. H., Graves, W. W., \& Conant, L. L. (2009). Where is the semantic system? A critical review and 
meta-analysis of 120 functional neuroimaging studies. Cerebral Cortex, 19, 2767-2796.

Bornkessel, I., Zysset, S., Friederici, A. D., von Cramon, D. Y., \& Schlesewsky, M. (2005). Who did what to whom? The neural basis of argument hierarchies during language comprehension. Neuroimage, 26, 221-233.

Brauer, J., \& Friederici, A. D. (2007). Functional neural networks of semantic and syntactic processes in the developing brain. Journal of Cognitive Neuroscience, 19, 1609-1623.

Conti-Ramsden, G., \& Durkin, K. (2012). Language development and assessment in the preschool period. Neuropsychology Review, 22, 384-401.

Cox, R. W., Reynolds, R. C., \& Taylor, P. A. (2016). AFNI and clustering: False positive rates redux. BioRxiv, 065862.

Davis, M. H., \& Rodd, J. M. (2011). Brain structures underlying lexical processing of speech: Evidence from brain imaging. In G. Gaskell \& P. Zwitserlood (Eds.), Lexical representation: A multidisciplinary approach (pp. 197-230). Berlin, Germany: Mouton de Gruyter.

den Ouden, D. B., Saur, D., Mader, W., Schelter, B., Lukic, S., Wali, E., et al. (2012). Network modulation during complex syntactic processing. Neuroimage, 59, 815-823.

Dibbets, P., Bakker, K., \& Jolles, J. (2006). Functional MRI of task switching in children with specific language impairment (SLI). Neurocase, 12, 71-79.

Dikker, S., Silbert, L. J., Hasson, U., \& Zevin, J. D. (2014). On the same wavelength: Predictable language enhances speakerlistener brain-to-brain synchrony in posterior superior temporal gyrus. Journal of Neuroscience, 34, 6267-6272.

Eklund, A., Nichols, T. E., \& Knutsson, H. (2016). Cluster failure: Why fMRI inferences for spatial extent have inflated falsepositive rates. Proceedings of the National Academy of Sciences, U.S.A., 113, 7900-7905.

Fedorenko, E., Hsieh, P. J., Nieto-Castañón, A., WhitfieldGabrieli, S., \& Kanwisher, N. (2010). New method for fMRI investigations of language: Defining ROIs functionally in individual subjects. Journal of Neurophysiology, 104, 1177-1194.

Fedorenko, E., Nieto-Castañon, A., \& Kanwisher, N. (2012). Lexical and syntactic representations in the brain: An fMRI investigation with multi-voxel pattern analyses. Neuropsychologia, 50, 499-513.

Friederici, A. D. (2002). Towards a neural basis of auditory sentence processing. Trends in Cognitive Sciences, 6, 78-84.

Friederici, A. D. (2012). The cortical language circuit: From auditory perception to sentence comprehension. Trends in Cognitive Sciences, 16, 262-268.

Friederici, A. D. (2018). The neural basis for human syntax: Broca's area and beyond. Current Opinion in Behavioral Sciences, 21, 88-92.

Friedrich, M., \& Friederici, A. D. (2005). Phonotactic knowledge and lexical-semantic processing in one-year-olds: Brain responses to words and nonsense words in picture contexts. Journal of Cognitive Neuroscience, 17, 1785-1802.

Gleitman, L. R., \& Wanner, E. (1982). Language acquisition: The state of the art (pp. 3-48). Cambridge, United Kingdom: Cambridge University Press.

Goucha, T., \& Friederici, A. D. (2015). The language skeleton after dissecting meaning: A functional segregation within Broca's Area. Neuroimage, 114, 294-302.

Hagoort, P., \& Indefrey, P. (2014). The neurobiology of language beyond single words. Annual Review of Neuroscience, 37, 347-362.

Haxby, J. V., Gobbini, M. I., Furey, M. L., Ishai, A., Schouten, J. L., \& Pietrini, P. (2001). Distributed and overlapping representations of faces and objects in ventral temporal cortex. Science, 293, 2425-2430.
Johnson, M. H. (2001). Functional brain development in humans. Nature Reviews Neuroscience, 2, 475-483.

Johnson, M. H. (2011). Interactive specialization: A domaingeneral framework for human functional brain development? Developmental Cognitive Neuroscience, 1, 7-21.

Karmiloff-Smith, A. (1998). Development itself is the key to understanding developmental disorders. Trends in Cognitive Sciences, 2, 389-398.

Kaufman, A. S., \& Kaufman, N. L. (2004). Kaufman Brief Intelligence Test-Second Edition. Bloomington, MN: Pearson.

Kim, J., Kim, Y. W., Park, C. I., Park, E. S., Kim, H. H., Lee, S. K., et al. (2006). Diffusion-tensor magnetic resonance imaging in children with language impairment. NeuroReport, 17, 1279-1282.

Knoll, L. J., Obleser, J., Schipke, C. S., Friederici, A. D., \& Brauer, J. (2012). Left prefrontal cortex activation during sentence comprehension covaries with grammatical knowledge in children. Neuroimage, 62, 207-216.

Kriegeskorte, N., Goebel, R., \& Bandettini, P. (2006). Information-based functional brain mapping. Proceedings of National Academy of Sciences, U.S.A., 103, 3863-3868.

Kuhl, P. K. (2010). Brain mechanisms in early language acquisition. Neuron, 67, 713-727.

Lewis-Peacock, J. A., \& Norman, K. A. (2013). Multi-voxel pattern analysis of fMRI data. In M. S. Gazzaniga \& G. R. Mangun (Eds.), The cognitive neurosciences (pp. 911-920). Cambridge, MA: MIT Press.

Masterson, J., Stuart, M., Dixon, M., \& Lovejoy, S. (2010). Children's printed word database: Continuities and changes over time in children's early reading vocabulary. British Journal of Psychology, 101, 221-242.

Mayes, A. K., Reilly, S., \& Morgan, A. T. (2015). Neural correlates of childhood language disorder: A systematic review. Developmental Medicine \& Child Neurology, 57, 706-717.

Mazaika, P., Hoeft, F., Glover, G. H., \& Reiss, A. L. (2009). Methods and software for fMRI analysis for clinical subjects. Paper presented at the 15th Annual Meeting of the Organization for Human Brain Mapping Human Brain Mapping Conference.

McNorgan, C., Chabal, S., O’Young, D., Lukic, S., \& Booth, J. R. (2015). Task dependent lexicality effects support interactive models of reading: A meta-analytic neuroimaging review. Neuropsychologia, 67, 148-158.

Morgan, A., Bonthrone, A., \& Liégeois, F. J. (2016). Brain basis of childhood speech and language disorders: Are we closer to clinically meaningful MRI markers? Current Opinion in Pediatrics, 28, 725-730.

Mumford, J. A., Turner, B. O., Ashby, F. G., \& Poldrack, R. A. (2012). Deconvolving BOLD activation in event-related designs for multivoxel pattern classification analyses. Neuroimage, 59, 2636-2643.

Nelson, D. L., McEvoy, C. L., \& Schreiber, T. A. (1998). The University of South Florida word association, rbyme, and word fragment norms [Database]. Retrieved from w3.usf. edu/FreeAssociation

Oberecker, R., \& Friederici, A. D. (2006). Syntactic event-related potential components in 24-month-olds' sentence comprehension. NeuroReport, 17, 1017-1021.

Oberecker, R., Friedrich, M., \& Friederici, A. D. (2005). Neural correlates of syntactic processing in two-year-olds Journal of Cognitive Neuroscience, 17, 1667-1678.

Obleser, J., \& Kotz, S. A. (2010). Expectancy constraints in degraded speech modulate the language comprehension network. Cerebral Cortex, 20, 633-640.

Pinker, S. (1984). Language learnability and language learning. Cambridge, MA: Harvard. 
Price, C. J. (2012). A review and synthesis of the first 20 years of PET and fMRI studies of heard speech, spoken language and reading. Neuroimage, 62, 816-847.

Rice, M. L. (2012). Toward epigenetic and gene regulation models of specific language impairment: Looking for links among growth, genes, and impairments. Journal of Neurodevelopmental Disorders, 4, 27.

Rice, M. L., \& Wexler, K. (2001). Rice/Wexler test of early grammatical impairment. San Antonio, TX: The Psychological Corporation.

Rodd, J. M., Vitello, S., Woollams, A. M., \& Adank, P. (2015). Localising semantic and syntactic processing in spoken and written language comprehension: An activation likelihood estimation meta-analysis. Brain and Language, 141, 89-102.

Seymour, H. N., Roeper, T., \& De Villiers, J. G. (2003). DELV: Diagnostic Evaluation of Language Variation: Screening test. San Antonio, TX: PsychCorp.

Sharp, D. J., Scott, S. K., \& Wise, R. J. (2004). Retrieving meaning after temporal lobe infarction: The role of the basal language area. Annals of Neurology, 56, 836-846.

Sirois, S., Spratling, M., Thomas, M. S., Westermann, G., Mareschal, D., \& Johnson, M. H. (2008). Précis of Neuroconstructivism: How the brain constructs cognition. Behavioral and Brain Sciences, 31, 321-331.

Skeide, M. A., Brauer, J., \& Friederici, A. D. (2014). Syntax gradually segregates from semantics in the developing brain. Neuroimage, 100, 106-111.

Skeide, M. A., Brauer, J., \& Friederici, A. D. (2016). Brain functional and structural predictors of language performance. Cerebral Cortex, 26, 2127-2139.
Skeide, M. A., \& Friederici, A. D. (2016). The ontogeny of the cortical language network. Nature Reviews Neuroscience, 17, 323-332.

Tager-Flusberg, H., \& Cooper, J. (1999). Present and future possibilities for defining a phenotype for specific language impairment. Journal of Speech, Language, and Hearing Research, 42, 1275-1278.

Wiig, E. H., Semel, E., \& Secord, W. A. (2013). Clinical Evaluation Language Fundamentals-Fifth Edition (CELF-5). San Antonio, TX: Pearson.

Wilke, M., Altaye, M., Holland, S. K., \& CMIND Authorship Consortium. (2017). CerebroMatic: A versatile toolbox for spline-based MRI template creation. Frontiers in Computational Neuroscience, 11, 5.

Wu, C. Y., Vissiennon, K., Friederici, A. D., \& Brauer, J. (2016). Preschoolers' brains rely on semantic cues prior to the mastery of syntax during sentence comprehension. Neuroimage, 126, 256-266.

Xia, M., Wang, J., \& He, Y. (2013). BrainNet Viewer: A network visualization tool for human brain connectomics. PLoS One, 8, e68910

Yang, Y. H., Marslen-Wilson, W. D., \& Bozic, M. (2017). Syntactic complexity and frequency in the neurocognitive language system. Journal of Cognitive Neuroscience, 29, 1605-1620.

Zaccarella, E., Schell, M., \& Friederici, A. D. (2017). Reviewing the functional basis of the syntactic Merge mechanism for language: A coordinate-based activation likelihood estimation meta-analysis. Neuroscience \& Biobehavioral Reviews, 80, 646-656. 
Copyright of Journal of Cognitive Neuroscience is the property of MIT Press and its content may not be copied or emailed to multiple sites or posted to a listserv without the copyright holder's express written permission. However, users may print, download, or email articles for individual use. 